\title{
Correction to: Prognostic value of estimated plasma volume in acute heart failure in three cohort studies
}

\author{
Masatake Kobayashi ${ }^{1,2} \cdot$ Patrick Rossignol $^{1,3}$ - João Pedro Ferreira ${ }^{1,4} \cdot$ Irene Aragão $^{4}$ Y Yuki Paku ${ }^{2}$ ' Yoichi Iwasaki ${ }^{2}$. \\ Masataka Watanabe $^{2} \cdot$ Marat Fudim $^{5,6} \cdot$ Kevin Duarte $^{1} \cdot$ Faiez Zannad $^{1,3} \cdot$ Nicolas Girerd $^{1,3}$
}

Published online: 6 November 2018

○) Springer-Verlag GmbH Germany, part of Springer Nature 2018

\section{Correction to: Clinical Research in Cardiology https://doi.org/10.1007/s00392-018-1385-1}

Unfortunately, an error occurred in the methods section of the abstract. The corrected text is given below.

Methods We retrospectively analyzed three cohort studies: 383 patients admitted at the Tokyo Medical University hospital, 165 patients admitted at the Centro Hospitalar do Porto and 164 patients admitted at the Nancy University Hospital (ICALOR study). ePVS at admission and at discharge as well as its change thereof were, respectively, calculated using the Duarte and Strauss formulas, both derived from hemoglobin and hematocrit ratios. Clinical variables including physical assessment, biological and echocardiographic parameters were recorded. The clinical outcome was a composite of re-hospitalization for worsening HF or all-cause mortality.

The original article has been corrected.

The original article can be found online at https://doi.org/10.1007/ s00392-018-1385-1.

Nicolas Girerd

n.girerd@chru-nancy.fr

1 INSERM, Centre d'Investigations Cliniques 1433, Centre d'Investigation Clinique Pierre Drouin, CHRU de Nancy, Inserm 1116 and INI-CRCT (Cardiovascular and Renal Clinical Trialists) F-CRIN Network, Institut lorrain du coeur et des, vaisseaux Louis Mathieu, Université de Lorraine, 4, rue du Morvan, 54500 Nancy, Vandoeuvre-Les-Nancy, France

2 Department of Cardiology, Tokyo Medical University, Tokyo, Japan

3 Département de Cardiologie, CHRU de Nancy, Nancy, France

4 Cardiovascular Research and Development Unit, Department of Physiology and Cardiothoracic Surgery, Faculty of Medicine, University of Porto, Porto, Portugal

5 Duke University Medical Center, Durham, NC, USA

6 Duke Clinical Research Institute, Durham, NC, USA 